\title{
Motion-induced energy shifts of a multilevel atom in a black-body radiation field
}

\author{
Huabing Cai ${ }^{1,2, a}{ }_{\mathbb{D}}$, Li-Gang Wang ${ }^{2, \mathrm{~b}}$ \\ ${ }^{1}$ Department of Physics, Anhui Normal University, Wuhu 241002, Anhui, China \\ 2 Department of Physics, Zhejiang University, Hangzhou 310027, Zhejiang, China
}

Received: 18 November 2021 / Accepted: 23 December 2021 / Published online: 13 January 2022

(C) The Author(s) 2022

\begin{abstract}
We investigate the influence of atomic uniform motion on radiative energy shifts of a multilevel atom when it interacts with black-body radiation. Our analysis reveals that the atomic energy shifts depend crucially on three factors: the temperature of black-body thermal radiation, atomic velocity, and atomic polarizability. In the low-temperature limit, the presence of atomic uniform motion always enhances the effect of the thermal field on the atomic energy shifts. However, in the high-temperature limit, the atomic uniform motion enhances the effect of the thermal field for an atom polarizable perpendicular to the atomic velocity but weakens it for an atom polarizable parallel to the atomic velocity. Our work indicates that the physical properties of atom-field coupling systems can in principle be regulated and controlled by the combined action of the thermal field and the atomic uniform motion.
\end{abstract}

\section{Introduction}

Quantum field theory predicts that quantum fields, even in the vacuum state, can fluctuate and thus may have rich content. Theory and experiment have adequately shown that vacuum fluctuations can give rise to some unusual physical consequences, such as spontaneous emission [1], Lamb shift [2,3], Casimir effect [4,5], and Casimir-Polder effect [6]. These effects are related to the interaction of matter (such as atoms) with quantum fields. Here we focus on the Lamb shift, i.e., the shift of the energy levels of an atom due to the perturbation of the quantum vacuum. Just like atomic spontaneous emission [1], the Lamb shift process can also be modified by external environments, for example, the presence of boundaries of quantum fields [7-14] or a change in the spacetime background [15-17]. Further, the presence of

\footnotetext{
a e-mail: caihuabing@126.com (corresponding author)

b e-mail: lgwang@zju.edu.cn
}

real photons instead of virtual photons of vacuum fluctuations can perturb the atomic radiative properties. Some works have found a temperature dependence in the shift of the energy levels for an atom immersed in a bath of black-body radiation [18-22]. It was found that the interaction between the atom and the black-body radiation produces two principal effects: the black-body radiation can both induce a dynamic Stark shift of the atomic energy levels and can drive transitions between atomic states [21]. Undoubtedly, the existence of boundaries can also modify thermal fluctuations. Zhu et al. considered the modification of atomic energy shifts by the presence of a conducting plane boundary in a thermal bath and the Casimir-Polder force between the atom and the conducting plane [12-14]. Some studies, inspired by the Unruh effect [23], have investigated the influence of the acceleration of the atom itself on the atomic radiative properties [24-27] using the formalism proposed by Dalibard, DupontRoc, and Cohen-Tannoudji (DDC) (see [28,29] for a separate discussion on the contributions of vacuum fluctuations and radiation reaction). When an atom is coupled to a massless quantum scalar field, the transition rates or the Lamb shift for a moving two-level atom with a constant acceleration $a$ in the Minkowski vacuum is the same as in the case for a static two-level atom in a thermal bath of the same field with the Unruh temperature $T=a /(2 \pi)$ [25]. The use of the DDC formalism also reveals that the contribution of vacuum fluctuations is modified by the atomic uniform acceleration, but the contribution of radiation reaction is completely unaffected. However, in contrast to the scalar field case, the effect of uniform acceleration on the atomic radiative properties is not totally equivalent to that of a thermal radiation field when a multilevel atom is coupled to a quantum electromagnetic field $[26,27]$. This does not imply a failure of the Unruh effect, because the Unruh effect just predicts that a uniformly accelerated observer in the Minkowski vacuum will observe a thermal bath (like black-body radiation) of the Rindler particles instead of the Minkowski particles. The 
abovementioned works and other related works have clearly indicated that the Lamb shift of atoms is strongly dependent on the boundary of the quantum fields, the type and the state of the quantum fields, the atomic motion, and the coupling type between the atom and the field.

In order to bring classical thermodynamics into accord with the principle of special relativity, some physicists are occupied in constructing a theory of relativistic thermodynamics. One key issue is the transformation laws of thermodynamic variables (such as temperature, entropy, heat) under Lorentz transformation. Initially, von Mosengil, Planck, and Einstein argued that the temperature of a moving body with a constant velocity $v$ is given by $T=T_{0} \sqrt{1-v^{2}}$, where $T_{0}$ is the temperature measured in the co-moving frame of reference. This indicates that a moving body appears cooler than it is at rest. However, after nearly half a century, this conclusion was challenged by Ott in 1963 [30], who proposed a different transformation form, $T=T_{0} / \sqrt{1-v^{2}}$. In other words, moving objects appear warmer. Since then, there has been great controversy over the theory of relativistic thermodynamics. In 1966, Landsberg presented yet another opinion that these thermodynamic variables are invariant under Lorentz transformation, in particular $T=T_{0}$ [31-33]. Some scholars tried to clean up the mess and controversy by developing a covariant theory of relativistic thermodynamics. Notably, van Kampen redefined a series of four-vector thermodynamic quantities and generalized the laws of thermodynamics to a covariant version [34]. Later, this theory was refined and developed by Israel [35]. There are other covariant theories and ideas from the perspective of statistical mechanics [36-40]. For a specific and detailed history, please see comments about this topic [41-46] and the references cited therein.

Black-body radiation, as a thermodynamic equilibrium system, has always played a pivotal role in the development of relativistic thermodynamics. Using the maximum entropy principle, Eberly and Kujawski derived a manifestly Lorentz-invariant generalization of the Gibbs's canonical density operator for free quantum electromagnetic fields [47]. The then-known results for the energy density, entropy, and other thermodynamic variables of black-body radiation were very simply re-derived. Contrary to the assertions of Ott and others, they believed that temperature may be conveniently considered as a purely rest-frame concept. Meanwhile, the related works of cosmic microwave background radiation (CMB) have contributed to the progress on the subject. CMB has been proved as a perfect black-body radiation with temperature $2.73 \mathrm{~K}$ in both theory and experiment. This boosts the study on the distribution of black-body cavity radiation in a moving frame of reference so as to estimate the velocity of the earth's motion with respect to the rest frame of CMB. From different perspectives, some authors obtained the same conclusion that the only effect of the uniform motion is to introduce an angle-dependent effective temperature, which replaces the rest-frame temperature [48-51]. This gives rise to the concept of "directional temperature" or "effective temperature," $T_{\text {eff }}=T_{0} \sqrt{1-v^{2}} /(1-v \cos \theta)$.

From a completely different point of view, using the relativistic quantum field theoretical methods, Costa and Matsas calculated the photon distribution detected by a uniformly moving Unruh-DeWitt detector immersed in the thermal bath of a massless quantum scalar field [52]. The moving particle detector, regarded as a special "thermometer" immersed in the thermal bath, probes a non-Planckian spectrum, which crucially depends on the detector's velocity. Thereby Landsberg and Matsas pointed out the nonexistence of a relativistic temperature transformation, because an observer moving in a heat reservoir cannot detect a black-body spectrum $[53,54]$. In fact, by integrating the Planckian spectrum with the directional temperature over the solid angle, the result of the Unruh-DeWitt detector can be exactly found without any appeal to quantum field theory. Nakamura argued that the inverse-temperature four-vector $\beta_{\mu}$ taken seriously in the van Kampen-Israel theory has a clearer meaning than the directional temperature [55]. The well-known expression with the directional temperature can be derived based on the inversetemperature four-vector. He also argued that the Boltzmann factor $e^{-\beta E}$ should be replaced with a more general form $e^{-\beta_{\mu} P^{\mu}}$, with $P^{\mu}$ being the energy-momentum of the system. Recently, in the framework of open quantum systems, Papadatos and Anastopoulos analyzed the thermodynamics of a uniformly moving quantum system interacting with a static thermal bath of the massless quantum scalar field [56]. Their analysis shows that the heat bath, in motion in the reference frame of the quantum system, is equivalent to a continuum of heat baths at rest with respect to the moving system, each with a different temperature. They then claimed that there is no unique rule for the Lorentz transformation of temperature. It should be noted here that although the photon number distribution or other physical quantities involving the moving black-body radiation can be accurately calculated in the rest frame of the black body or through various approaches, the description of the thermodynamic properties of the moving black-body radiation still remains controversial. As can be seen, relativistic thermodynamics has always been, and will continue to be, a controversial topic.

Inspired by the work of Costa and Matsas [52], Cai investigated the transition process of a uniformly moving atom coupled to a bath of the thermal quantum electromagnetic field [57]. It was shown that the atomic transition rates depend crucially on the atomic velocity, the temperature of the thermal bath, and the atomic polarizability. Again, the moving atom as a photon detector also probes a non-Planckian spectrum. Notably, because the electric field of the moving black-body radiation is anisotropic, the spectrum function recorded by the atom polarizable perpendicular to the atomic velocity is quite different from the case for the atom polarizable parallel 
to the atomic velocity. The abovementioned works clearly imply that the thermal state of quantum fields is relativistic and observer-dependent. It is clear that this characteristic of black-body radiation can affect the transition processes of a uniformly moving atom. Quite naturally, one should ask whether it can also cause the shift of atomic levels. In fact, we recently investigated the influence of this characteristic on the energy shift of a two-level atom coupled to a thermal bath of a massless quantum scalar field [58]. The study of this simple model preliminarily revealed that the moving thermal radiation field can shift the atomic levels in a way quite different from that of the static ones. In this paper, we generalize this toy model into the case of a real multilevel atom in electric dipole interaction with a quantum electromagnetic field. It is assumed that initially the atom in a certain stationary state makes a uniform linear motion and that the quantum field is in the thermal state at a finite temperature (i.e., the blackbody radiation field). Using the DDC formalism [28,29], we separately calculate the contributions of thermal fluctuations and radiation reaction on the atomic energy shifts and explore how they are respectively modified by the coexistence of the thermal bath and the atomic uniform motion. This may contribute to further exploration on controlling and adjusting the physical properties of an atom-field coupling system. On the other hand, by examining the effect of the relativity of thermal states on the atomic radiative properties in detail, it may contribute to the further understanding of various theories of relativistic thermodynamics. The natural units $\hbar=c=1$ and $k_{B}=1$ are adopted throughout the paper.

\section{The DDC formula for the energy shift of a multilevel atom coupled to the quantum electromagnetic field}

We consider the interacting system of a multilevel atom coupled to a quantum electromagnetic field. The time evolution of the whole system with respect to the atomic proper time $\tau$ can be governed by the total Hamiltonian $H(\tau)=$ $H_{A}(\tau)+H_{F}(\tau)+H_{I}(\tau)$. $H_{A}(\tau)$ is the Hamiltonian operator of the atom with a set of discrete levels,

$H_{A}(\tau)=\sum_{n} \omega_{n} \sigma_{n n}(\tau)$,

where $\sigma_{n n}=|n\rangle\langle n|$, and $\omega_{n}$ corresponds to the energy of the stationary state $|n\rangle . H_{F}(\tau)$ is the Hamiltonian operator of the quantum electromagnetic field, expressed as

$H_{F}(\tau)=\sum_{\mathbf{k} \lambda} \omega_{\mathbf{k}} a_{\mathbf{k} \lambda}^{\dagger} a_{\mathbf{k} \lambda} \frac{\mathrm{d} t}{\mathrm{~d} \tau}$,

where $a_{\mathbf{k} \lambda}\left(a_{\mathbf{k} \lambda}^{\dagger}\right)$ is the annihilation (creation) operator for a photon with wave vector $\mathbf{k}$ and polarization $\lambda$, and $\omega_{\mathbf{k}}$ is the photon's energy. The atom-field coupling is described by the interaction Hamiltonian $H_{I}(\tau)$, and here we consider in the multipolar coupling scheme the electric dipole interaction [59]

$H_{I}(\tau)=-e \mathbf{r}(\tau) \cdot \mathbf{E}\left(X_{A}(\tau)\right)$,

where $e$ is the electron charge, $e \mathbf{r}(\tau)$ denotes the atomic electric dipole moment operator, and $\mathbf{E}(X)$ is the electric field operator. It should be noted that the coupling is effective only on the spacetime trajectory $X_{A}(\tau)$ of the atom. Assume that the atom is initially prepared in the stationary state $|b\rangle$ and the quantum field is in an arbitrary quantum state $|\Phi\rangle$. Since the DDC formalism has been widely employed to investigate the radiative properties of atoms in different situations in order to separately discuss the contributions of quantum fluctuations and radiation reaction [12-16,24-27], here we immediately arrive at the general expression of quantum fluctuations and radiation reaction contributions to the energy shift of the multilevel atom,

$\left(\delta E_{b}\right)_{\mathrm{qf}}=-i e^{2} \int_{\tau_{0}}^{\tau} \mathrm{d} \tau^{\prime}\left(C_{i j}^{F}\right)_{\Phi}\left(X_{A}(\tau), X_{A}\left(\tau^{\prime}\right)\right)\left(\chi_{i j}^{A}\right)_{b}\left(\tau, \tau^{\prime}\right)$,
$\left(\delta E_{b}\right)_{\mathrm{rr}}=-i e^{2} \int_{\tau_{0}}^{\tau} \mathrm{d} \tau^{\prime}\left(\chi_{i j}^{F}\right)_{\Phi}\left(X_{A}(\tau), X_{A}\left(\tau^{\prime}\right)\right)\left(C_{i j}^{A}\right)_{b}\left(\tau, \tau^{\prime}\right)$.

The symmetric correlation function $\left(C_{i j}^{F}\right)_{\Phi}$ and the linear susceptibility $\left(\chi_{i j}^{F}\right)_{\Phi}$ of the quantum electromagnetic field in the state $|\Phi\rangle$ are defined as follows:

$\left(C_{i j}^{F}\right)_{\Phi}\left(X_{A}(\tau), X_{A}\left(\tau^{\prime}\right)\right)=\frac{1}{2}\left\langle\Phi\left|\left\{\mathrm{E}_{i}\left(X_{A}(\tau)\right), \mathrm{E}_{j}\left(X_{A}\left(\tau^{\prime}\right)\right)\right\}\right| \Phi\right\rangle$,

$\left(\chi_{i j}^{F}\right)_{\Phi}\left(X_{A}(\tau), X_{A}\left(\tau^{\prime}\right)\right)=\frac{1}{2}\left\langle\Phi\left|\left[\mathrm{E}_{i}\left(X_{A}(\tau)\right), \mathrm{E}_{j}\left(X_{A}\left(\tau^{\prime}\right)\right)\right]\right| \Phi\right\rangle$.

$\left(C_{i j}^{A}\right)_{b}\left(\tau, \tau^{\prime}\right)$ and $\left(\chi_{i j}^{A}\right)_{b}\left(\tau, \tau^{\prime}\right)$ are the statistical functions of the atom in the stationary state $|b\rangle$. They are not dependent on the trajectory of the atom, and their explicit forms are given by

$$
\begin{aligned}
\left(C_{i j}^{A}\right)_{b}\left(\tau, \tau^{\prime}\right)= & \frac{1}{2}\left\langle b\left|\left\{\mathrm{r}_{i}(\tau), \mathrm{r}_{j}\left(\tau^{\prime}\right)\right\}\right| b\right\rangle \\
= & \frac{1}{2} \sum_{d}\left[\left\langle b\left|\mathrm{r}_{i}(0)\right| d\right\rangle\left\langle d\left|\mathrm{r}_{j}(0)\right| b\right\rangle e^{i \omega_{b d} \Delta \tau}\right. \\
& \left.+\left\langle b\left|\mathrm{r}_{j}(0)\right| d\right\rangle\left\langle d\left|\mathrm{r}_{i}(0)\right| b\right\rangle e^{-i \omega_{b d} \Delta \tau}\right], \\
\left(\chi_{i j}^{A}\right)_{b}\left(\tau, \tau^{\prime}\right)= & \frac{1}{2}\left\langle b\left|\left[\mathrm{r}_{i}(\tau), \mathrm{r}_{j}\left(\tau^{\prime}\right)\right]\right| b\right\rangle \\
= & \frac{1}{2} \sum_{d}\left[\left\langle b\left|\mathrm{r}_{i}(0)\right| d\right\rangle\left\langle d\left|\mathrm{r}_{j}(0)\right| b\right\rangle e^{i \omega_{b d} \Delta \tau}\right. \\
& \left.-\left\langle b\left|\mathrm{r}_{j}(0)\right| d\right\rangle\left\langle d\left|\mathrm{r}_{i}(0)\right| b\right\rangle e^{-i \omega_{b d} \Delta \tau}\right],
\end{aligned}
$$

where the notations $\Delta \tau=\tau-\tau^{\prime}$ and $\omega_{b d}=\omega_{b}-\omega_{d}$ are adopted, and the sum extends over a complete set of atomic states. The result of energy shifts can be quite different for different trajectories $X_{A}(\tau)$ and quantum states of field $|\Phi\rangle$. We will discuss a specific case in the following. 


\section{The case for a uniformly moving atom in black-body radiation}

Now we apply the above formalism to calculate the energy shifts of a uniformly moving atom immersed in a black-body radiation field at a finite temperature $T$. The atom moves at a constant velocity $v$ along a certain direction, for example, the $z$ direction, and then its world line is denoted in Cartesian coordinates as

$X_{A}(\tau)=\left(\gamma \tau, x_{0}, y_{0}, z_{0}+v \gamma \tau\right)$,

where $\left(x_{0}, y_{0}, z_{0}\right)$ is the atomic initial spatial coordinates, and the relativistic factor $\gamma=1 / \sqrt{1-v^{2}}$. The thermal state of the quantum field is described by the density operator $\rho=e^{-\beta H_{F}}$ with the inverse temperature $\beta=1 / T$. By the formula $\langle\beta|G| \beta\rangle=\operatorname{tr}(\rho G) / \operatorname{tr}(\rho)$ with $G$ being an arbitrary field operator, the two-point correlation function of the electromagnetic four-vector potential $A_{\mu}(X)$ in the thermal state $|\beta\rangle$ is given by

$$
\begin{aligned}
& \left\langle\beta\left|A_{\mu}(X) A_{\nu}\left(X^{\prime}\right)\right| \beta\right\rangle=-\eta_{\mu \nu} \int d^{3} \mathbf{k} \\
& \times\left[\frac{e^{\omega / T}}{e^{\omega / T}-1} u_{\mathbf{k}}(X) u_{\mathbf{k}}^{*}\left(X^{\prime}\right)+\frac{1}{e^{\omega / T}-1} u_{\mathbf{k}}^{*}(X) u_{\mathbf{k}}\left(X^{\prime}\right)\right],
\end{aligned}
$$

where $\eta_{\mu \nu}=(1,-1,-1,-1)$ and

$u_{\mathbf{k}}(X)=\sqrt{\frac{1}{2 \omega(2 \pi)^{3}}} e^{i k_{1} x} e^{i k_{2} y} e^{i k_{3} z} e^{-i \omega t}$.

Due to the relation $E_{i}(X)=\partial_{i} A_{0}(X)-\partial_{0} A_{i}(X)$, after insertion into the atomic trajectory $(10)$ and some calculations and simplifications, the correlation functions of the electric field in the instantaneous reference frame of the atom are found to be [57]

$$
\begin{gathered}
\left\langle\beta\left|E_{i}\left(X_{A}(\tau)\right) E_{j}\left(X_{A}\left(\tau^{\prime}\right)\right)\right| \beta\right\rangle=\delta_{i j} \frac{1}{3 \pi} \int_{0}^{\infty} \mathrm{d} \omega \omega^{3} \\
\times\left[e^{-i \omega \Delta \tau}\left(1+f_{i}(\omega, \beta, v)\right)+e^{i \omega \Delta \tau} f_{i}(\omega, \beta, v)\right],
\end{gathered}
$$

where the functions $f_{i}(\omega, \beta, v)$ with $i=1,2,3$ are defined as

$$
\begin{aligned}
f_{1}(\omega, \beta, v)= & \frac{3}{4} \int_{0}^{\pi} \mathrm{d} \theta\left(1-\frac{1}{2} \sin ^{2} \theta\right) \frac{\sin \theta}{e^{\omega \beta \gamma(1-v \cos \theta)}-1} \\
=- & \frac{3}{4} \sum_{k=1}^{\infty} \frac{1}{k^{3} \omega^{3} \beta^{3} v^{3} \gamma^{3}} \\
& {\left[\left(1+k \omega \beta v \gamma+k^{2} \omega^{2} \beta^{2} v^{2} \gamma^{2}\right) e^{-k \omega \beta(1+v) \gamma}\right.} \\
& \left.-\left(1-k \omega \beta v \gamma+k^{2} \omega^{2} \beta^{2} v^{2} \gamma^{2}\right) e^{-k \omega \beta(1-v) \gamma}\right], \\
f_{2}(\omega, \beta, v)= & f_{1}(\omega, \beta, v), \\
f_{3}(\omega, \beta, v)= & \frac{3}{4} \int_{0}^{\pi} \mathrm{d} \theta\left(1-\cos ^{2} \theta\right) \frac{\sin \theta}{e^{\omega \beta \gamma(1-v \cos \theta)}-1}
\end{aligned}
$$

$$
\begin{aligned}
= & \frac{3}{2} \sum_{k=1}^{\infty} \frac{1}{k^{3} \omega^{3} \beta^{3} v^{3} \gamma^{3}}\left[(1+k \omega \beta v \gamma) e^{-k \omega \beta(1+v) \gamma}\right. \\
& \left.-(1-k \omega \beta v \gamma) e^{-k \omega \beta(1-v) \gamma}\right] .
\end{aligned}
$$

Here we have used the identity $\sum_{k=1}^{\infty} e^{-k a}=\frac{1}{e^{a}-1}(a>0)$ to give two different forms of $f_{i}(\omega, \beta, v)$. Then the symmetric correlation function and the linear susceptibility of the field are easily given by

$$
\begin{aligned}
\left(C_{i j}^{F}\right)_{\beta}\left(X_{A}(\tau), X_{A}\left(\tau^{\prime}\right)\right)= & \delta_{i j} \frac{1}{3 \pi} \int_{0}^{\infty} \mathrm{d} \omega \omega^{3}\left(1+2 f_{i}(\omega, \beta, v)\right) \\
& \times\left(e^{-i \omega \Delta \tau}+e^{i \omega \Delta \tau}\right), \\
\left(\chi_{i j}^{F}\right)_{\beta}\left(X_{A}(\tau), X_{A}\left(\tau^{\prime}\right)\right)= & \delta_{i j} \frac{1}{3 \pi} \int_{0}^{\infty} \mathrm{d} \omega \omega^{3}\left(e^{-i \omega \Delta \tau}-e^{i \omega \Delta \tau}\right) .
\end{aligned}
$$

Substituting (17) and (18) into Eqs. (4) and (5), and assuming sufficiently long time evolution $\tau-\tau_{0} \rightarrow \infty$, we then obtain the contributions of thermal fluctuations and radiation reaction to the energy shift of the atom in the state $|b\rangle$,

$$
\begin{aligned}
\left(\delta E_{b}\right)_{\mathrm{tf}}= & \frac{e^{2}}{3 \pi} \sum_{d}|\langle b|\mathbf{r}(0)| d\rangle|^{2} \\
& \times \int_{0}^{\infty} \mathrm{d} \omega \omega^{3}\left(1+2 \alpha_{i} f_{i}(\omega, \beta, v)\right) P \\
& \left(\frac{1}{\omega+\omega_{b d}}-\frac{1}{\omega-\omega_{b d}}\right), \\
\left(\delta E_{b}\right)_{\mathrm{rr}}= & -\frac{e^{2}}{3 \pi} \sum_{d}|\langle b|\mathbf{r}(0)| d\rangle|^{2} \\
& \times \int_{0}^{\infty} \mathrm{d} \omega \omega^{3} P\left(\frac{1}{\omega+\omega_{b d}}+\frac{1}{\omega-\omega_{b d}}\right),
\end{aligned}
$$

where we have used the notation $\alpha_{i}=\left|\left\langle b\left|\mathrm{r}_{i}(0)\right| d\right\rangle\right|^{2} /$ $|\langle b|\mathbf{r}(0)| d\rangle|^{2}$, and $P$ denotes the Cauchy principal value. Compared with the case for a static atom in a black-body radiation field [18-21,26], it can be seen that the contribution of thermal fluctuations is modified by the atomic inertial motion, as the factor $2 \alpha_{i} f_{i}(\omega, \beta, v)$ is a nonthermal term (i.e., not $\frac{2}{e^{\omega \beta}-1}$ ). However, the contribution of radiation reaction is the same as in the case for an inertial atom in vacuum or for a static atom in a thermal bath [26], which is independent of both the temperature and the atomic velocity. Adding up the two contributions, we can obtain the total energy shift

$$
\begin{aligned}
\delta E_{b}= & \frac{2 e^{2}}{3 \pi} \sum_{d}|\langle b|\mathbf{r}(0)| d\rangle|^{2} \\
& \times \int_{0}^{\infty} \mathrm{d} \omega \omega^{3} P\left(\left[\alpha_{i} f_{i}(\omega, \beta, v)\right] \frac{1}{\omega+\omega_{b d}}\right. \\
& \left.-\left[1+\alpha_{i} f_{i}(\omega, \beta, v)\right] \frac{1}{\omega-\omega_{b d}}\right) .
\end{aligned}
$$


The energy shift can be divided into two parts, $\delta E_{b}=$ $\left(\delta E_{b}\right)_{0}+\left(\delta E_{b}\right)_{T, v}$, where

$$
\begin{aligned}
\left(\delta E_{b}\right)_{0}= & -\frac{2 e^{2}}{3 \pi} \sum_{d}|\langle b|\mathbf{r}(0)| d\rangle|^{2} \\
& \times \int_{0}^{\infty} \mathrm{d} \omega \omega^{3} P\left(\frac{1}{\omega-\omega_{b d}}\right)
\end{aligned}
$$

and

$$
\begin{aligned}
\left(\delta E_{b}\right)_{T, v}= & \frac{2 e^{2}}{3 \pi} \sum_{d}|\langle b|\mathbf{r}(0)| d\rangle|^{2} \\
& \times \int_{0}^{\infty} \mathrm{d} \omega \omega^{3} \alpha_{i} f_{i}(\omega, \beta, v) P \\
& \left(\frac{1}{\omega+\omega_{b d}}-\frac{1}{\omega-\omega_{b d}}\right) .
\end{aligned}
$$

Here, $\left(\delta E_{b}\right)_{0}$ refers to the known Lamb shift term caused by vacuum fluctuations. $\left(\delta E_{b}\right)_{T, v}$ is exactly the modification of the energy shift caused by the combination of the black-body radiation field and the atomic uniform motion, which depends on the temperature of the thermal field, the atomic velocity, and the atomic polarizability. Since the renormalized $\left(\delta E_{b}\right)_{0}$ is a constant, we focus only on the term $\left(\delta E_{b}\right)_{T, v}$.

In particular, for the isotropic polarization, i.e., $\alpha_{i}=$ $\left(\frac{1}{3}, \frac{1}{3}, \frac{1}{3}\right)$, the result becomes

$$
\begin{aligned}
\left(\delta E_{b}\right)_{T, v}= & \frac{2 e^{2}}{3 \pi} \sum_{d}|\langle b|\mathbf{r}(0)| d\rangle|^{2} \\
& \times \int_{0}^{\infty} \mathrm{d} \omega \omega^{3} h(\omega, \beta, v) P \\
& \left(\frac{1}{\omega+\omega_{b d}}-\frac{1}{\omega-\omega_{b d}}\right),
\end{aligned}
$$

where

$$
\begin{aligned}
h(\omega, \beta, v) & =\frac{1}{2} \int_{0}^{\pi} \mathrm{d} \theta \frac{\sin \theta}{e^{\omega \beta \gamma(1-v \cos \theta)}-1} \\
& =\sum_{k=1}^{\infty} \frac{\sqrt{1-v^{2}}}{2 k \omega \beta v}\left[e^{-\frac{k \omega \beta(1-v)}{\sqrt{1-v^{2}}}}-e^{-\frac{k \omega \beta(1+v)}{\sqrt{1-v^{2}}}}\right] \\
& =\frac{\sqrt{1-v^{2}}}{2 \omega \beta v} \ln \left(\frac{1-e^{-\frac{\omega \beta(1+v)}{\sqrt{1-v^{2}}}}}{1-e^{-\frac{\omega \beta(1-v)}{\sqrt{1-v^{2}}}}}\right) .
\end{aligned}
$$

The function $h(\omega, \beta, v)$ is exactly proportional to the known spectrum function of the black-body radiation in the moving reference frame already obtained in previous studies [48-52].

It is often difficult to eliminate this principal integral in (22). However, approximate analytical results can be obtained in some limited cases. Meanwhile, numerical results can be given in order to analyze the behavior of the atomic energy shifts. In the following, we will examine them in detail.

\section{Analytical analysis and numerical analysis}

Choosing the dimensionless variables $\lambda_{0}=\omega_{b d} \beta$ and $\lambda=$ $\omega \beta$, one has

$$
\left(\delta E_{b}\right)_{T, v}=\frac{2 e^{2}}{3 \pi} \sum_{d}|\langle b|\mathbf{r}(0)| d\rangle|^{2} \omega_{b d}^{3} F\left(\lambda_{0}, v\right)
$$

where the dimensionless factor

$$
\begin{aligned}
F\left(\lambda_{0}, v\right)= & \frac{1}{\lambda_{0}^{3}} \int_{0}^{\infty} d \lambda \lambda^{3} \alpha_{i} f_{i}(\lambda, v) P \\
& \times\left(\frac{1}{\lambda+\lambda_{0}}-\frac{1}{\lambda-\lambda_{0}}\right) .
\end{aligned}
$$

In the limit of low temperature, i.e., $\left|\hbar \omega_{b d}\right| \gg k_{B} T$ holds for all $\omega_{b d}$, the energy shift becomes approximately

$$
\begin{aligned}
& \left(\delta E_{b}\right)_{T, v}^{\text {perp }} \approx \frac{2 e^{2}}{3 \pi} \sum_{d}|\langle b|\mathbf{r}(0)| d\rangle|^{2} \omega_{b d}^{3} \\
& \times\left[\frac{2 \pi^{4}\left(1+v^{2}\right)}{15\left(1-v^{2}\right)}\left(\frac{T}{\omega_{b d}}\right)^{4}+\frac{16 \pi^{6}\left(1+\frac{17}{5} v^{2}+\frac{2}{5} v^{4}\right)}{63\left(1-v^{2}\right)^{2}}\left(\frac{T}{\omega_{b d}}\right)^{6}\right]
\end{aligned}
$$

for the polarizability $\alpha_{i}=(1,0,0)$ or $\alpha_{i}=(0,1,0)$,

$$
\begin{aligned}
& \left(\delta E_{b}\right)_{T, v}^{\mathrm{para}} \approx \frac{2 e^{2}}{3 \pi} \sum_{d}|\langle b|\mathbf{r}(0)| d\rangle|^{2} \omega_{b d}^{3} \\
& \times\left[\frac{2 \pi^{4}}{15}\left(\frac{T}{\omega_{b d}}\right)^{4}+\frac{16 \pi^{6}\left(1+v^{2} / 5\right)}{63\left(1-v^{2}\right)}\left(\frac{T}{\omega_{b d}}\right)^{6}\right]
\end{aligned}
$$

for the polarizability $\alpha_{i}=(0,0,1)$, and

$$
\begin{aligned}
& \left(\delta E_{b}\right)_{T, v}^{\text {iso }} \approx \frac{2 e^{2}}{3 \pi} \sum_{d}|\langle b|\mathbf{r}(0)| d\rangle|^{2} \omega_{b d}^{3} \\
& \times\left[\frac{2 \pi^{4}\left(1+v^{2} / 3\right)}{15\left(1-v^{2}\right)}\left(\frac{T}{\omega_{b d}}\right)^{4}+\frac{16 \pi^{6}\left(1+2 v^{2}+\frac{1}{5} v^{4}\right)}{63\left(1-v^{2}\right)^{2}}\left(\frac{T}{\omega_{b d}}\right)^{6}\right]
\end{aligned}
$$

for the polarizability $\alpha_{i}=\left(\frac{1}{3}, \frac{1}{3}, \frac{1}{3}\right)$. In contrast to Eq. (12) in [21], the presence of the atomic uniform motion clearly modifies the level shift of the atom in thermal radiation. We find that for an atom polarizable parallel to the direction of atomic motion, the leading term is not affected and the second term is enhanced by the factor $\frac{1+v^{2} / 5}{1-v^{2}}$. However, for an atom polarizable perpendicular to the direction of atomic motion, both the leading term and the second term are amplified. As a result, the energy shift for an atom polarizable perpendicular to the direction of atomic motion is slightly more obvious than that for an atom polarizable parallel to the direction of atomic motion. In short, in the low-temperature limit, the uniform motion always enhances the effect of the thermal field on the atomic energy shifts no matter what the atomic polarizability is. As shown in Eq. (12) in [21], the energy shift of a static atom in the low temperature limit is proportional to $T^{4}$. Replacing the temperature $T$ by the effective temperature 
$T_{\text {eff }}=T \sqrt{1-v^{2}} /(1-v \cos \theta)$ in the result of the static atom and integrating it over the solid angle with different density factors, i.e., $\left(1-\frac{1}{2} \sin ^{2} \theta\right),\left(1-\cos ^{2} \theta\right)$ and 1 in Eqs. (14)-(16) and (24) respectively for atoms with different polarizabilities, the above results (27)-(29) can be recovered. Due to the form of the atom-field interaction Hamiltonian (3), the moving atom polarized along the $x$ (or $z$ ) direction perceives only the $x$ (or $z$ ) component of the electric field. The dependence of the results on the atomic polarizabilities is exactly due to the anisotropy of the two-point correlation functions for the spatial components of the electric field (see Eq. (13) with Eqs. (14)-(16)). This absolutely reflects the fact that the black-body radiation seen by the moving observer is anisotropic due to the relativistic Doppler effect.

In the high-temperature limit, i.e., $\left|\hbar \omega_{b d}\right| \ll k_{B} T$ holds for all $\omega_{b d}$, one has

$$
\begin{aligned}
& \left(\delta E_{b}\right)_{T, v}^{\text {perp }} \approx \frac{2 e^{2}}{3 \pi} \sum_{d}|\langle b|\mathbf{r}(0)| d\rangle|^{2} \omega_{b d}^{3} \\
& \quad \times\left[\frac{3\left(1-v^{2}\right) \ln \frac{1-v}{1+v}+6 v}{4 v^{3}}\left(-\frac{\pi^{2}}{3}\right)\left(\frac{T}{\omega_{b d}}\right)^{2}\right]
\end{aligned}
$$

for the polarizability $\alpha_{i}=(1,0,0)$ or $\alpha_{i}=(0,1,0)$,

$$
\begin{aligned}
& \left(\delta E_{b}\right)_{T, v}^{\text {para }} \approx \frac{2 e^{2}}{3 \pi} \sum_{d}|\langle b|\mathbf{r}(0)| d\rangle|^{2} \omega_{b d}^{3} \\
& \times\left[\frac{\left(v^{2}-1\right)\left(6 v+3 \ln \frac{1-v}{1+v}\right)}{2 v^{3}}\left(-\frac{\pi^{2}}{3}\right)\left(\frac{T}{\omega_{b d}}\right)^{2}\right]
\end{aligned}
$$

for the polarizability $\alpha_{i}=(0,0,1)$, and

$$
\begin{gathered}
\left(\delta E_{b}\right)_{T, v}^{\text {iso }} \approx \frac{2 e^{2}}{3 \pi} \sum_{d}|\langle b|\mathbf{r}(0)| d\rangle|^{2} \omega_{b d}^{3} \\
\times\left[\left(-\frac{\pi^{2}}{3}\right)\left(\frac{T}{\omega_{b d}}\right)^{2}\right]
\end{gathered}
$$

for the polarizability $\alpha_{i}=\left(\frac{1}{3}, \frac{1}{3}, \frac{1}{3}\right)$. Notably, the energy shift of the isotropically polarizable atom is not affected by the uniform motion. In contrast to Eq. (11) in [21], it is easy to prove that the presence of the uniform motion always enhances the action of the thermal field on the energy shift for an atom polarizable perpendicular to the direction of atomic motion, but weakens that for an atom polarizable parallel to the direction of atomic motion. As shown in Eq. (11) in [21], the energy shift of a static atom in the high-temperature limit is proportional to $T^{2}$. Again, replacing the temperature $T$ by the effective temperature $T_{\text {eff }}=T \sqrt{1-v^{2}} /(1-v \cos \theta)$ in the result of the static atom and integrating it over the solid angle with different density factors, the above results (30)-(32) can be recovered. The insensitivity to the atomic velocity for the result of the isotropically polarizable atom is due to the fact that the average temperature squared defined as $\left\langle T_{\mathrm{eff}}^{2}\right\rangle=\frac{1}{4 \pi} \int T_{\mathrm{eff}}^{2}(v, \theta) \mathrm{d} \Omega$ is independent of $v$.
When the atomic velocity is small as compared with the light velocity $(|v| \ll 1)$, one has

$$
\begin{aligned}
f_{1}(\omega, \beta, v) & =f_{2}(\omega, \beta, v) \approx \frac{1}{e^{\omega \beta}-1}+\tilde{f}_{1}(\omega, \beta) v^{2}, \\
f_{3}(\omega, \beta, v) & \approx \frac{1}{e^{\omega \beta}-1}+\tilde{f}_{3}(\omega, \beta) v^{2}, \\
h(\omega, \beta, v) & \approx \frac{1}{e^{\omega \beta}-1}+\tilde{h}(\omega, \beta) v^{2},
\end{aligned}
$$

where we have defined the functions

$$
\begin{aligned}
\tilde{f}_{1}(\omega, \beta)= & \frac{2}{5}\left(\frac{\omega \beta}{e^{\omega \beta}-1}+\frac{\omega \beta}{2}-\frac{5}{4}\right)\left(\frac{1}{e^{\omega \beta}-1}+1\right) \\
& \times\left(\frac{\omega \beta}{e^{\omega \beta}-1}\right), \\
\tilde{f}_{3}(\omega, \beta)= & \frac{1}{5}\left(\frac{\omega \beta}{e^{\omega \beta}-1}+\frac{\omega \beta}{2}-\frac{5}{2}\right)\left(\frac{1}{e^{\omega \beta}-1}+1\right) \\
& \times\left(\frac{\omega \beta}{e^{\omega \beta}-1}\right), \\
\tilde{h}(\omega, \beta)= & \frac{1}{3}\left(\frac{\omega \beta}{e^{\omega \beta}-1}+\frac{\omega \beta}{2}-\frac{3}{2}\right)\left(\frac{1}{e^{\omega \beta}-1}+1\right) \\
& \times\left(\frac{\omega \beta}{e^{\omega \beta}-1}\right) .
\end{aligned}
$$

Then the energy shift reduces to

$$
\left(\delta E_{b}\right)_{T, v}=\left(\delta E_{b}\right)_{T}+\left(\delta E_{b}\right)_{v},
$$

where

$$
\begin{aligned}
\left(\delta E_{b}\right)_{T}= & \frac{2 e^{2}}{3 \pi} \sum_{d}|\langle b|\mathbf{r}(0)| d\rangle|^{2} \\
& \times \int_{0}^{\infty} \mathrm{d} \omega \omega^{3} \frac{1}{e^{\omega \beta}-1} P\left(\frac{1}{\omega+\omega_{b d}}-\frac{1}{\omega-\omega_{b d}}\right)
\end{aligned}
$$

and

$$
\begin{aligned}
\left(\delta E_{b}\right)_{v}= & {\left[\frac{2 e^{2}}{3 \pi} \sum_{d}|\langle b|\mathbf{r}(0)| d\rangle|^{2}\right.} \\
& \left.\times \int_{0}^{\infty} \mathrm{d} \omega \omega^{3} \alpha_{i} \tilde{f}_{i}(\omega, \beta) P\left(\frac{1}{\omega+\omega_{b d}}-\frac{1}{\omega-\omega_{b d}}\right)\right] v^{2} .
\end{aligned}
$$

Here, $\left(\delta E_{b}\right)_{T}$ refers to the result of a static multilevel atom immersed in a bath of thermal radiation [21], and $\left(\delta E_{b}\right)_{v}$ refers to the sole modification due to the slow motion of the atom. $\left(\delta E_{b}\right)_{v}$ is proved to be convergent and nonzero; then the modification of the energy shift due to the slow motion of the atom is proportional to $v^{2}$. The slow motion of the atom can enhance or weaken the action of the thermal bath, as $\left(\delta E_{b}\right)_{v}$ can be positive or negative depending on the temperature of the thermal radiation, the atomic level distribution, and the atomic polarizability.

The dependence of the atomic energy shift on the temperature and the velocity is entirely contained in the function $F\left(\lambda_{0}, v\right)$. For general values of $T$ and $v$, here we give some 


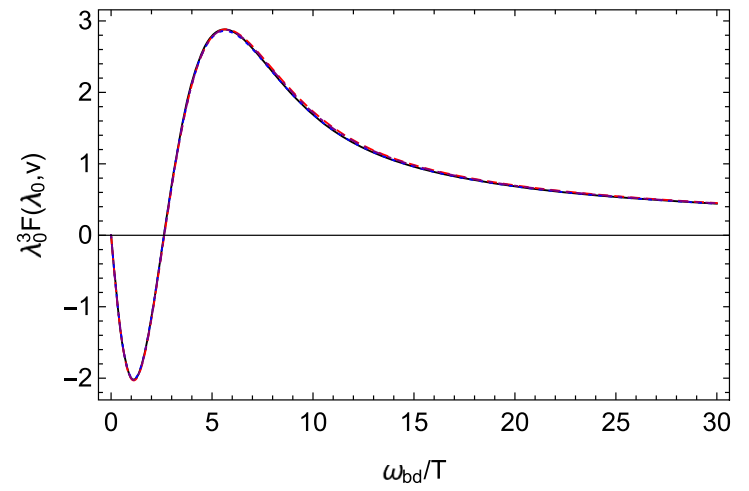

(a)

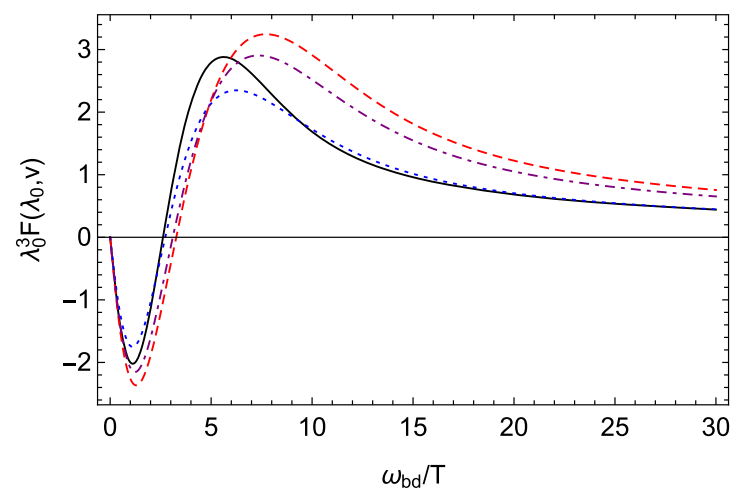

(c)

Fig. 1 The behavior of the function $\lambda_{0}^{3} F\left(\lambda_{0}, v\right)$ with the increase in $\lambda_{0}$. The dashed, dotted, and dot-dashed lines refer to the cases for an atom polarizable perpendicular to the atomic velocity, parallel to the atomic velocity, and isotropically, respectively. As a contrast, the black solid

numerical results. In Fig. 1, for better presentation, we depict the function $\lambda_{0}^{3} F\left(\lambda_{0}, v\right)$ instead of $F\left(\lambda_{0}, v\right)$, just as in the reference [21]. Here we only show the case for positive $\omega_{b d}$, and the result for negative $\omega_{b d}$ can be obtained by the relation $\left(-\lambda_{0}\right)^{3} F\left(-\lambda_{0}, v\right)=-\lambda_{0}^{3} F\left(\lambda_{0}, v\right)$. As can be seen, the function is always negative in the zone of small $\omega_{b d} / T$, but it is always positive in the zone of big $\omega_{b d} / T$. This means that when the temperature is fixed, those states $|d\rangle$ close to the atomic state $|b\rangle$ tend to draw the atomic level closer to themselves, whereas those states $|d\rangle$ distant from the atomic state $|b\rangle$ tend to repel it. This characteristic is also tenable in the case of a static atom in thermal radiation [21]. Although the presence of uniform motion can reinforce or weaken this feature, it does not reverse it. By Fig. 2 we depict the behavior of the function $F\left(\lambda_{0}, v\right)$ with the increase in the magnitude of the atomic velocity. Notably, the behavior of $F\left(\lambda_{0}, v\right)$ is quite different for different polarizabilities. The graph of the function can also be of various shapes for different cases of $\omega_{b d} / T$. The function $F\left(\lambda_{0}, v\right)$ always ends up returning to 0 when $v \rightarrow 1$. This means that the energy shifts of ultra-

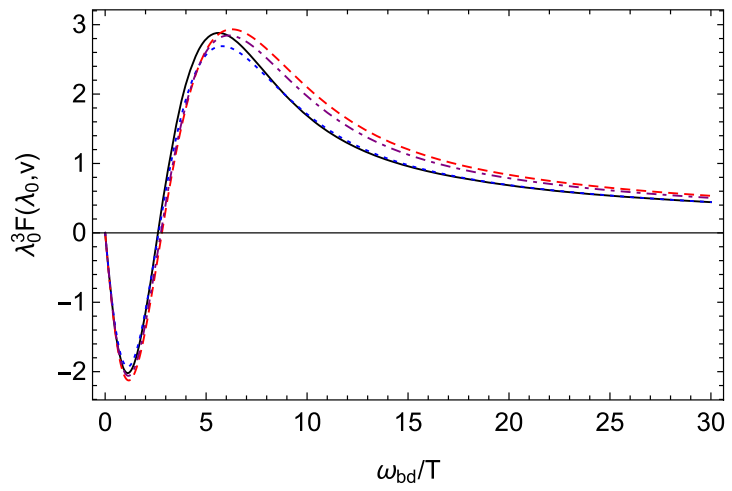

(b)

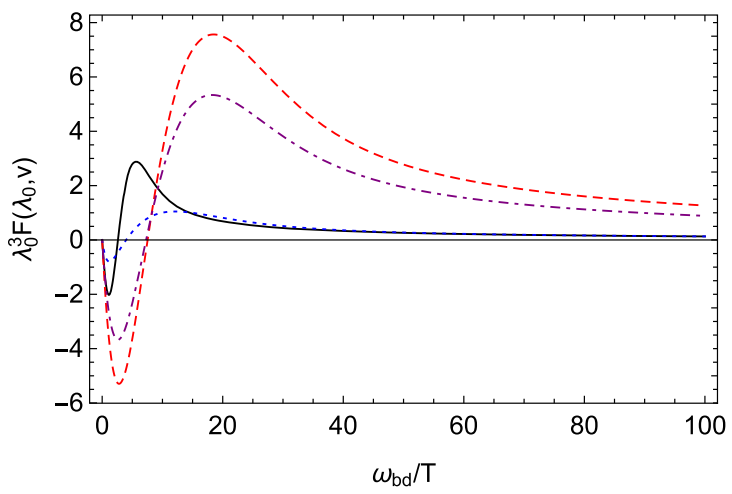

(d)

line refers to the case for a static atom in the same thermal radiation $(v=0)$. a The case for $v=0.1, \mathbf{b}$ the case for $v=0.3, \mathbf{c}$ the case for $v=0.5, \mathbf{d}$ the case for $v=0.9$

relativistic atoms are unaffected by the thermal radiation as if the atom were in vacuum. This characteristic also appears in the investigation of the transition rates for a uniformly moving Unruh-DeWitt detector or atom in a thermal radiation field [52,57]. Costa and Matsas in [52] explicitly point out that this can be understood physically on the basis of energy conservation. In this respect, if the relativistic transformation law of temperature exists, Einstein-Planck transformation law, $T=T_{0} \sqrt{1-v^{2}}$, may be more reasonable than that of Ott. However, we should note that for the atom polarizable perpendicular to the atomic velocity or polarizable isotropically, the fall to 0 is swift as $v \rightarrow 1$. Only for the atom polarizable parallel to the atomic velocity does the line fall smoothly to 0 . Thus, for high velocity (for example, the velocity is greater than $v=0.9$ ), the value of $F\left(\lambda_{0}, v\right)$ can be still considerable. On the whole, in the zone of high velocity, the deviation from the case of $v=0$ due to the effect of uniform motion is prominent. By contrast, with the curves for a static atom in thermal radiation (the transverse solid lines), it is clearly shown that the presence of uniform motion can 


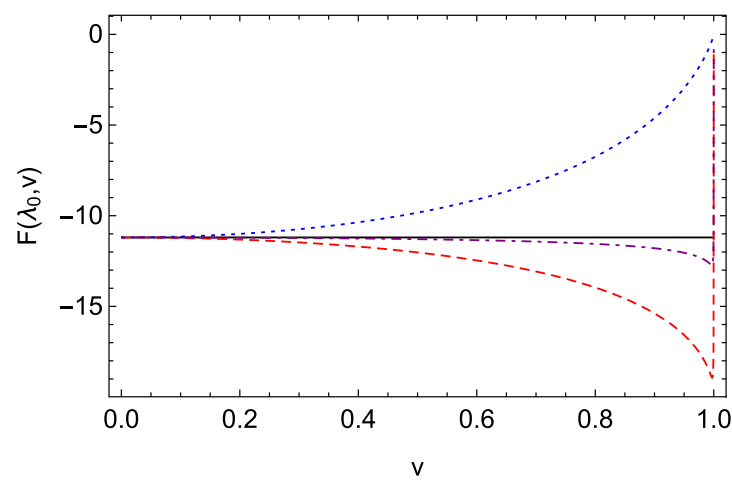

(a)

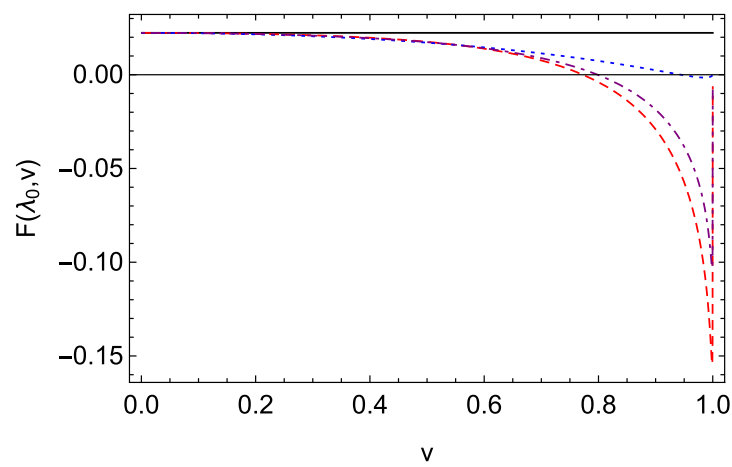

(c)

Fig. 2 The behavior of the function $F\left(\lambda_{0}, v\right)$ with the increase in $v$. The dashed, dotted, and dot-dashed lines refer to the cases for an atom polarizable perpendicular to the atomic velocity, parallel to the atomic velocity, and isotropically, respectively. As a contrast, the transverse

enhance or weaken the effect of the thermal radiation at different levels, depending on the specific values of $T, v$, and the atomic polarizability. Moreover, the sign of $F\left(\lambda_{0}, v\right)$ can vary with $v$, and thus the uniform motion can reverse the sign of the atomic energy shift in appropriate conditions. It should be noted that the numerical images here also confirm the correctness of the previous analytical results.

\section{Conclusions}

Using the DDC formalism, we have separately calculated the contributions of thermal fluctuations and radiation reaction to the energy shifts of a uniformly moving multilevel atom in a black-body radiation field. The result shows that the contribution of thermal fluctuations is strongly affected by the combined action of thermal radiation and atomic uniform motion. However, that of radiation reaction is completely impervious. This is because the linear susceptibility of the electric field defined in Eq. (7) is not affected by either the uniform motion or the state of the field (see Eq. (18)). The total energy shifts

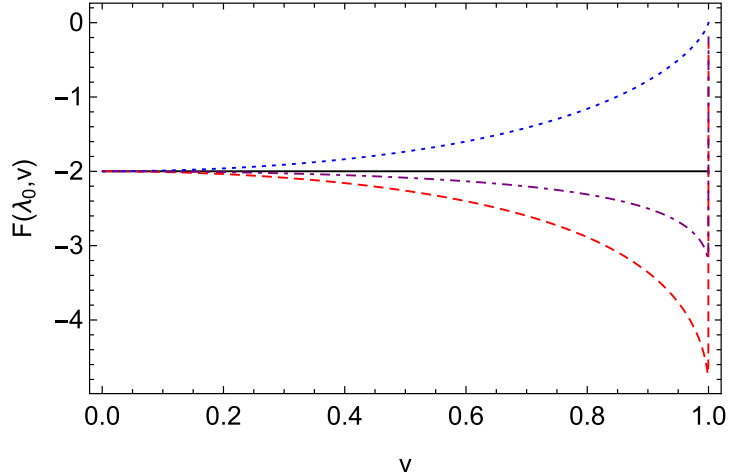

(b)

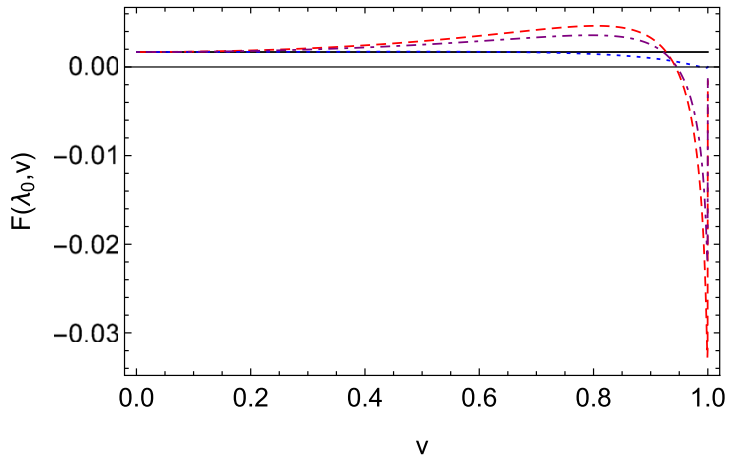

(d)

solid line refers to the case for a static atom in the same thermal radiation $(v=0)$. a The case for $\omega_{b d} / T=0.5$, $\mathbf{b}$ the case for $\omega_{b d} / T=1$, c the case for $\omega_{b d} / T=5, \mathbf{d}$ the case for $\omega_{b d} / T=10$

depend crucially on the temperature of the thermal radiation, the atomic velocity, and the atomic polarizability. Notably, the behavior of energy shifts can be quite different when the atom has different polarizabilities. The dependence on the atomic polarizability reflects the anisotropy of the moving black-body radiation as seen by the atom regarded as a special photon detector. In the low-temperature limit, the presence of the atomic uniform motion always enhances the action of the thermal bath on the atomic energy shifts. The impact of uniform motion for an atom polarizable perpendicular to the direction of atomic motion is stronger than that in the case for an atom polarizable parallel to the direction of atomic motion. However, the situation is different in the high-temperature regime. The presence of uniform motion always enhances the action of the thermal field on the energy shift for an atom polarizable perpendicular to the direction of atomic motion but weakens that for the atom polarizable parallel to the direction of atomic motion. These peculiarities for the energy shifts in the low or high regime are in sharp contrast to the case for a uniformly moving two-level atom coupled to a thermal bath of a massless quantum scalar 
field [58]. Moreover, the modification of the energy shift due solely to the slow motion of the atom is always proportional to $v^{2}$. Our results indicate that the moving black body as seen by the atom indeed shifts the atomic levels in a way quite different from that of the static one. More specifically, by adjusting the abovementioned influencing parameters, the atomic uniform motion can raise or lower the atomic energy levels. We thus conclude that the relativity of the thermal state of a quantum electromagnetic field can both affect the transitions between atomic states [57] and cause the shift of the atomic levels. Theoretically, the radiative properties of the atom-field coupling system can be regulated by the combination of the thermal field and the atomic uniform motion. This may have theoretical value and guiding significance for some areas of atomic physics and optics. In fact, our results can also be obtained in the co-moving frame of reference of the atom by using the manifestly Lorentz-invariant generalization of the Gibbs's canonical density operator derived in [47], $\rho=e^{-V_{\mu} P^{\mu} /\left(k_{B} T\right)}$, where $V_{\mu} / k_{B} T$ is exactly the inverse-temperature four-vector $\beta_{\mu}$. The inverse-temperature four-vector can be regarded as a combination of the temperature and the four-velocity. This reminds us of the energymomentum four-vector of a particle in relativistic mechanics as a combination of the mass and the four-velocity, $p_{\mu}=m V_{\mu}$, where $m$ is the rest mass of the particle. Just like the relation between the rest mass and the relativistic mass of a particle, $M=m V_{0}=\frac{m}{\sqrt{1-v^{2}}}$ associated with Einstein's mass-energy relation $E=M c^{2}$, one can also define the "relativistic temperature" of the thermodynamic equilibrium system, $T_{v}=T / V_{0}=T \sqrt{1-v^{2}}$, which is in line with the transformation law of von Mosengil, Planck, and Einstein. Of course, it would be better to hold the view that the temperature is the parameter just defined in the rest frame of the system like the rest mass. We tend to argue that the moving thermal radiation field, at least for the massless quantum scalar field and the quantum electromagnetic field, should be described by the density operator $\rho=e^{-\beta_{\mu} P^{\mu}}$ instead of $\rho=e^{-\beta H_{F}}$.

Acknowledgements This work is supported by Anhui Provincial Natural Science Foundation (Grant No. 2008085QA25). This research is also supported by Zhejiang Provincial Natural Science Foundation of China under Grant No. LD18A040001, and the grant by National Key Research and Development Program of China (No. 2017YFA0304202); it was also supported by the National Natural Science Foundation of China (Grant No. 11974309).

Data Availability Statement This manuscript has no associated data or the data will not be deposited. [Authors' comment: All data generated or analysed during this study are included in this published article.]

Open Access This article is licensed under a Creative Commons Attribution 4.0 International License, which permits use, sharing, adaptation, distribution and reproduction in any medium or format, as long as you give appropriate credit to the original author(s) and the source, provide a link to the Creative Commons licence, and indicate if changes were made. The images or other third party material in this article are included in the article's Creative Commons licence, unless indicated otherwise in a credit line to the material. If material is not included in the article's Creative Commons licence and your intended use is not permitted by statutory regulation or exceeds the permitted use, you will need to obtain permission directly from the copyright holder. To view a copy of this licence, visit http://creativecomm ons.org/licenses/by/4.0/.

Funded by SCOAP ${ }^{3}$.

\section{References}

1. H. Walther, B.T.H. Varcoe, B.G. Englert, T. Becker, Cavity quantum electrodynamics. Rep. Prog. Phys. 69, 1325 (2006)

2. W.E. Lamb, R.C. Retherford, Fine structure of the hydrogen atom by a microwave method. Phys. Rev. 72, 241 (1947)

3. M.O. Scully, A.A. Svidzinsky, The Lamb shift-yesterday, today, and tomorrow. Science 328, 1239-1241 (2010)

4. H.B.G. Casimir, On the attraction between two perfectly conducting plates. Proc. Kon. Ned. Akad. Wet. 51, 793 (1948)

5. M. Bordag, G.L. Klimchitskaya, U. Mohideen, V.M. Mostepanenko, Advances in the Casimir effect (Oxford University Press, Oxford, 2009)

6. H.B.G. Casimir, D. Polder, The influence of retardation on the London-van der Waals forces. Phys. Rev. 73, 360 (1948)

7. G. Barton, Quantum electrodynamics of spinless particles between conducting plates. Proc. R. Soc. Lond. Ser. A 320, 251 (1970)

8. D. Meschede, W. Jhe, E.A. Hinds, Radiative properties of atoms near a conducting plane: an old problem in a new light. Phys. Rev. A 41, 1587 (1990)

9. W. Jhe, QED level shifts of atoms between two mirrors. Phys. Rev. A 43, 5795 (1991)

10. E.A. Hinds, V. Sandoghdar, Cavity QED level shifts of simple atoms. Phys. Rev. A 43, 398 (1991)

11. Z. Mohammadi, F. Kheirandish, Energy-level shifts and the decay rate of an atom in the presence of a conducting wedge. Phys. Rev. A 92, 062118 (2015)

12. W. She, H. Yu, Z. Zhu, Casimir-Polder interaction between an atom and an infinite boundary in a thermal bath. Phys. Rev. A 81, 012108 (2010)

13. Z. Zhu, H. Yu, Modification of energy shifts of atoms by the presence of a boundary in a thermal bath and the Casimir-Polder force. Phys. Rev. A 79, 032902 (2009)

14. Z. Zhu, H. Yu, B. Wang, Temperature-dependent Casimir-Polder forces on polarizable molecules. Rev. A 86, 052508 (2012)

15. W. Zhou, H. Yu, Lamb shift in de Sitter spacetime. Phys. Rev. D 82, 124067 (2010)

16. W.Zhou, H. Yu, Lamb shift for static atoms outside a Schwarzschild black hole. Phys. Rev. D 82, 104030 (2010)

17. W. Zhou, H. Yu, Can spacetime curvature induced corrections to Lamb shift be observable? J. High Energy Phys. 2012, 172 (2012)

18. J.E. Walsh, Possibility of thermal corrections in radiative level-shift determination. Phys. Rev. Lett. 27, 208 (1971)

19. G. Barton, Temperature dependence of the Lamb shift. Phys. Rev. A 5, 468 (1972)

20. P.L. Knight, Effects of external fields on the Lamb shift. J. Phys. A 5, 417 (1972)

21. J.. W. Farley, W..H. Wing, Accurate calculation of dynamic Stark shifts and depopulation rates of Rydberg energy levels induced by blackbody radiation. Hydrogen, helium, and alkali-metal atoms. Phys. Rev. A 23, 2397 (1981)

22. U.D. Jentschura, M. Haas, Reexamining blackbody shifts for hydrogenlike ions. Phys. Rev. A 78, 042504 (2008) 
23. L.C.B. Crispino, A. Higuchi, G.E.A. Matsas, The Unruh effect and its applications. Rev. Mod. Phys. 80, 787 (2008)

24. J. Audretsch, R. Müller, Spontaneous excitation of an accelerated atom: the contributions of vacuum fluctuations and radiation reaction. Phys. Rev. A 50, 1755 (1994)

25. J. Audretsch, R. Müller, Radiative energy shifts of an accelerated two-level system. Phys. Rev. A 52, 629 (1995)

26. R. Passante, Radiative level shifts of an accelerated hydrogen atom and the Unruh effect in quantum electrodynamics. Phys. Rev. A 57, 1590 (1998)

27. Z. Zhu, H. Yu, S. Lu, Spontaneous excitation of an accelerated hydrogen atom coupled with electromagnetic vacuum fluctuations. Phys. Rev. D 73, 107501 (2006)

28. J. Dalibard, J. Dupont-Roc, C. Cohen-Tannoudji, Vacuum fluctuations and radiation reaction: identification of their respective contributions. J. Phys. (Paris) 43, 16171617 (1982)

29. J. Dalibard, J. Dupont-Roc, C. Cohen-Tannoudji, Dynamics of a small system coupled to a reservoir: reservoir fluctuations and selfreaction. J. Phys. (Paris) 45, 637 (1984)

30. H. Ott, Lorentz-Transformation der Wärme und der Temperatur. Z. Phys. 175, 70 (1963)

31. P.T. Landsberg, Special relativistic thermodynamics. Proc. Phys. Soc. London 89, 1007 (1966)

32. P.T. Landsberg, Does a moving body appear cool? Nature 212, 571 (1966)

33. P.T. Landsberg, K.A. Johns, A relativistic generalization of thermodynamics. Nuovo Cim. B 52, 28 (1967)

34. N.G. Van Kampen, Relativistic thermodynamics of moving systems. Phys. Rev. 173, 295 (1968)

35. W. Israel, Nonstationary irreversible thermodynamics: a causal relativistic theory. Ann. Phys. 100, 310-331 (1976)

36. T.S. Bíró, P. Ván, About the temperature of moving bodies. Europhys. Lett. 89, 30001 (2010)

37. G. Ares de Parga, B. López-Carrera, Relativistic statistical mechanics vs. relativistic thermodynamics. Entropy 13, 1664-1693 (2011)

38. C. Farías, V.A. Pinto, P.S. Moya, What is the temperature of a moving body? Sci. Rep. 7, 1-9 (2017)

39. A.S. Parvan, Lorentz transformations of the thermodynamic quantities. Ann. Phys. 401, 130-138 (2019)

40. X. Hao, S. Liu, L. Zhao, Relativistic transformations for temperature and chemical potential via kinetic theory (2021). arXiv:2105.07313
41. C.K. Yuen, Lorentz transformation of thermodynamic quantities. Am. J. Phys. 38, 246 (1970)

42. D. Ter Haar, H. Wergeland, Thermodynamics and statistical mechanics in the special theory of relativity. Phys. Rep. 1, 31 (1971)

43. C. Liu, Is there a relativistic thermodynamics? A case study of the meaning of special relativity. Stud. Hist. Philos. Sci. 25, 983 (1994)

44. T.K. Nakamura, Three views of a secret in relativistic thermodynamics. Prog. Theor. Phys. 128, 463-475 (2012)

45. K. Derakhshani, Black body radiation in moving frames (2019). arXiv: 1908.08599

46. K.T. McDonald, Temperature and special relativity (2020)

47. J.H. Eberly, A. Kujawski, Relativistic statistical mechanics and blackbody radiation. Phys. Rev. 155, 10 (1967)

48. R.N. Bracewell, E.K. Conklin, An observer moving in the $3^{\circ} \mathrm{K}$ radiation field. Nature 219, 1343 (1968)

49. P.J.E. Peebles, D.T. Wilkinson, Comment on the anisotropy of the primeval fireball. Phys. Rev. 174, 2168 (1968)

50. C.V. Heer, R.H. Kohl, Theory for the measurement of the earth's velocity through the $3^{\circ} \mathrm{K}$ cosmic radiation. Phys. Rev. 174, 1611 (1968)

51. G.R. Henry, R.B. Feduniak, J.E. Silver, M.A. Peterson, Distribution of blackbody cavity radiation in a moving frame of reference. Phys. Rev. 176, 1451 (1968)

52. S.S. Costa, G.E.A. Matsas, Temperature and relativity. Phys. Lett. A 209, 155 (1995)

53. P.T. Landsberg, G.E.A. Matsas, Laying the ghost of the relativistic temperature transformation. Phys. Lett. A 223, 401 (1996)

54. P.T. Landsberg, G.E.A. Matsas, The impossibility of a universal relativistic temperature transformation. Phys. A 340, 92 (2004)

55. T.K. Nakamura, Lorentz transform of black-body radiation temperature. Europhys. Lett. 88, 20004 (2009)

56. N. Papadatos, C. Anastopoulos, Relativistic quantum thermodynamics of moving systems. Phys. Rev. D 102, 085005 (2020)

57. H. Cai, Velocity effect on the stimulated transition process of a multilevel atom in a thermal bath. Eur. Phys. J. C 81, 673 (2021)

58. H. Cai, L. Wang, Energy shift of a uniformly moving two-level atom in a thermal bath. PRA (2021)

59. G. Compagno, R. Passante, F. Persico, Atom-field interactions and dressed atoms (Cambridge University Press, Cambridge, 1995) 